\title{
A Novel Multimedia Streaming System for Urban Rail Environments Using Wi-Fi Peer- to-Peer Technology
}

Poderys, Justas; Farooq, Jahanzeb; Soler, José

Published in:

Proceedings of 2018 IEEE 87th Vehicular Technology Conference

Link to article, DOI:

10.1109/VTCSpring.2018.8417799

Publication date:

2018

Document Version

Peer reviewed version

Link back to DTU Orbit

Citation (APA):

Poderys, J., Farooq, J., \& Soler, J. (2018). A Novel Multimedia Streaming System for Urban Rail Environments Using Wi-Fi Peer-to-Peer Technology. In Proceedings of 2018 IEEE 87th Vehicular Technology Conference IEEE. https://doi.org/10.1109/VTCSpring.2018.8417799

\section{General rights}

Copyright and moral rights for the publications made accessible in the public portal are retained by the authors and/or other copyright owners and it is a condition of accessing publications that users recognise and abide by the legal requirements associated with these rights.

- Users may download and print one copy of any publication from the public portal for the purpose of private study or research.

- You may not further distribute the material or use it for any profit-making activity or commercial gain

- You may freely distribute the URL identifying the publication in the public portal 


\title{
A Novel Multimedia Streaming System for Urban Rail Environments Using Wi-Fi Peer-to-Peer Technology
}

\author{
Justas Poderys $^{1}$, Jahanzeb Farooq ${ }^{1,2}$, and Jose Soler ${ }^{1}$ \\ ${ }^{1}$ Department of Photonics Engineering \\ Technical University of Denmark \\ Kongens Lyngby, Denmark \\ \{juspo, jafar, joss $\} @$ fotonik.dtu.dk \\ ${ }^{2}$ Siemens $A / S$ \\ Ballerup, Denmark \\ jahanzeb.farooq@siemens.com
}

\begin{abstract}
The amount of streaming multimedia data delivered to mobile devices is growing at a high rate. Research shows that a large number of daily commuters stream audio and video to their mobile devices during their travels. This makes urban rail environments a suitable platform for delivering entertainment, information and advertisement multimedia using novel delivery techniques. In order to do so, the system presented in this paper utilizes the unused bandwidth of a Communications-Based Train Control link to transmit multimedia to urban trains. Once on the train, multimedia is distributed to passenger devices using Wi-Fi Peer-to-Peer (P2P) technology. Such a multimedia distribution system can be deployed incrementally, as it can function concurrently with Wi-Fi connections already available in a number of trains. This paper presents the results obtained by emulating multimedia streaming in an urban rail use-case. Namely, it evaluates the received streaming multimedia quality parameters when new users arrive or existing users are replaced during the train stops.
\end{abstract}

Index Terms-CBTC, train communication, multimedia, streaming, peer-to-peer, Wi-Fi Direct

\section{INTRODUCTION}

Mobile devices data usage is growing at a high rate. According to an industry report [1], data usage by smartphones is growing at $42 \%$ Compounded Annual Growth Rate (CAGR). Of all data transferred to mobile devices, the amount of video and audio streaming data is growing at 50\% and 34\% CAGR, respectively. Furthermore, between $30 \%$ and $40 \%$ of smartphone users stream multimedia data during their commute [1]. The widespread usage of smartphones to receive streaming multimedia allows the deployment of new services, such as location-based information, entertainment, and advertising.

At present, urban rail passengers receive the Internet connectivity from commercial cellular networks directly, or via the onboard Wi-Fi connectivity [2]. However, during peak usage periods, like sports matches or live shows, the cellular network can get overwhelmed, reducing the quality of received multimedia. As the amount of data consumed by smartphone users is expected to continue increasing, urban rail serves as a valuable candidate to deploy technologies that can reduce the load on the cellular networks.
In order to deliver high-quality multimedia to passengers, two tasks must be accomplished. First, the multimedia data must be delivered to the train car. This paper describes a system that delivers streaming multimedia to urban trains by utilizing the unused capacity of a Communications-Based Train Control (CBTC) radio communication link [3]. Second, once delivered to the train car, it must be distributed among the users. As urban trains contain a large number of users located in a limited area, it allows for the use of a peer-to-peer wireless networking technology to do so. Work presented here uses the Wi-Fi Peer-to-Peer (P2P, also known as Wi-Fi Direct) [4] technology along with the Peer-to-Peer Streaming Peer Protocol (PPSPP) [5] clients running in the passenger devices. Using Wi-Fi P2P to establish connections between the passengers offers several advantages. First, it utilizes the physical (PHY) layer of the conventional IEEE 802.11 Wi-Fi technology, and it is already widely available on modern mobile devices. Second, it allows to use peer-to-peer connectivity without disconnecting from an Access-Point (AP) of a conventional Wi-Fi network. This allows for incremental deployment of WiFi P2P based data distribution in urban trains that already have conventional Wi-Fi connectivity available.

The rest of the paper is structured as follows. Section II presents related work in this field. The proposed multimedia streaming system and its component are presented in section III. The set-up of experimental system evaluation and evaluation results are described in section IV. Finally, section V presents conclusions.

\section{RELATED WORK}

The topic of delivering streaming multimedia in a public transport environment has been investigated in several works already. However, these works focus on the quality of data connection when delivering data to e.g. a train [6], [7] or a metro [8], and not on the quality of the delivered multimedia. For example, the authors of [7] investigate the network performance of Mobile IP in scenarios involving high-speed trains as a train performs handovers between base stations. 
Their performance evaluation focuses on average throughput and packet loss at the transport layer. Similarly, the work presented in [6] measures the performance of an in-train multimedia distribution system based on Wi-Fi multicast by using packet-loss rate as a performance indicator. Finally, the work presented in [8] aims to improve the quality of the received video in a passenger information system in a metro by optimizing the handover algorithm by employing fountain codes for error correction. However, the work uses Signalto-Noise Ratio (SNR) as an approximation for multimedia streaming quality without considering multimedia streaming continuity. In contrast to the above mentioned works, our work focuses specifically on the Quality-of-Service (QoS) of the delivered multimedia (described in more details in section IV), and not on the network layer parameters, such as average throughput or packet loss.

A large body of knowledge exists analyzing the Wi-Fi Peerto-Peer (P2P) technology and its specific features. A thorough Wi-Fi P2P technical overview, including experimental evaluation of group formation delays and energy efficiency, is presented in [9]. Several papers in the field investigate the possibility of implementing opportunistic networks using WiFi P2P technology. The authors of [10] use experiments with up to three mobile phones to estimate the time required to form concurrent communication groups.

There are several papers considering multimedia streaming over Wi-Fi P2P. A mobile peer-to-peer video streaming solution is also presented in [11]. The authors propose to use Wi-Fi P2P as an alternative to cellular data connections when peers are located nearby. Similar to the work presented here, the authors propose to use mesh-based overlay streaming protocol. However, in contrast to the work presented in this paper, the streaming multimedia source in [11] is a centralized streaming server. Research presented in [12] use analytical and simulation analysis to show that Wi-Fi P2P can be used to offload LTE-based cellular connections between the devices in close proximity.

\section{IN-TRAIN MULTIMEDIA STREAMING}

Presently, two most widespread methods to deliver data connectivity to the urban rail passengers are (1) by providing a cellular connection directly to each passenger device, or (2) by providing a cellular connection to a gateway device in the train car which is then used to provide connectivity to passengers' devices using Wi-Fi technology, as shown in Fig. 1. The cellular connection in these two methods can be based on e.g. 3G/4G [2], WiMAX [6], or IEEE 802.11p (WAVE) [8].

The first method, shown in shown in Fig. 1.A, offers data connectivity directly to a a user's device. However, in order for it to work, users (or the train) must be inside the cellular network's coverage area. In addition, interruptions in the data connection can be experienced as a fast moving train roams from one base station to another [13], [14]. Furthermore, since streaming multimedia is a bandwidth-intensive application, it can incur significant data transfer charges due to the usage of cellular connection.
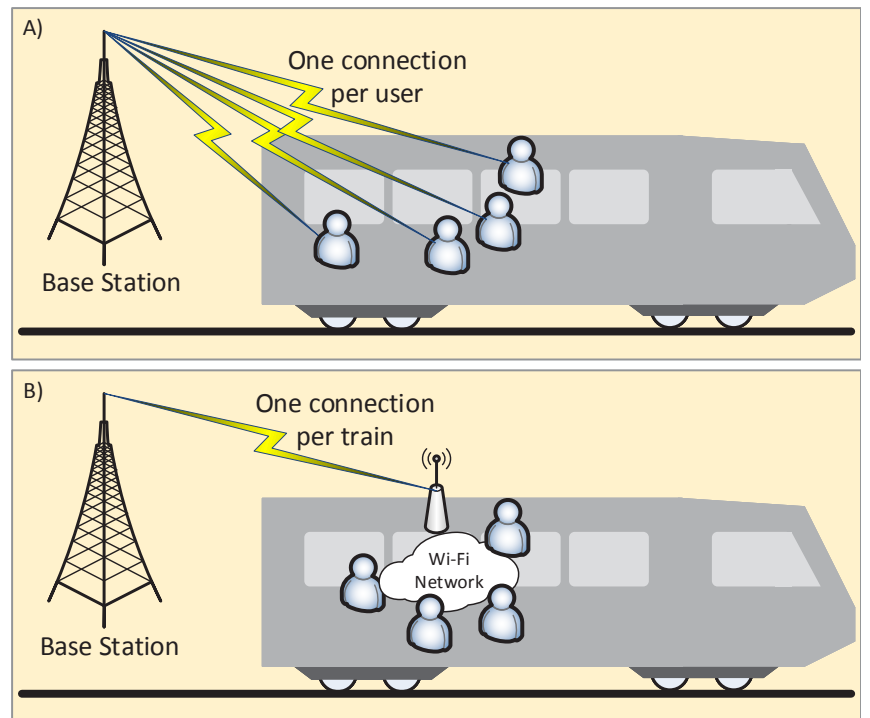

Fig. 1. Methods of delivering data connectivity to the passengers: (A) using a direct cellular connection for each user, (B) using one cellular connection for the train that is shared with other users using Wi-Fi.

The second connectivity method, shown in Fig. 1.B, involves providing data connection to a gateway device inside a train, which is then shared among the passengers using conventional Wi-Fi technology. Nonetheless, this method likewise requires cellular network coverage. In addition, providing a secure data connection is problematic when using conventional Wi-Fi. As per the Wi-Fi standard [15], secure communication can be enabled by encrypting the transmissions either by using a pre-shared key or a user certificate. As these methods are impractical for mass deployment, Wi-Fi connections in public transport are typically unencrypted.

A potential solution in these scenarios is to reduce the downlink connection load. During periods of peak usage, such as sports events or live shows, multiple users will stream the same multimedia content. By doing so, users increase the load on the downlink connection to the train with multiple copies of identical data. By using technologies that reduce the load on the downlink connection, a larger number of users can be served with high-quality multimedia.

In order to address the above challenges, this paper proposes a novel method of streaming multimedia to the train passenger. The overview of the system is shown in Fig. 2. In order to distribute multimedia to the train passengers, it is delivered to the train using the available bandwidth of the CBTC connection, as described in further details in section III-A. The selection of radio technology used to implement the CBTC connection is out of the scope of this paper. However, it is assumed the chosen technology provides enough spare capacity to deliver multimedia data. Once data is delivered to the train, it is distributed among the passengers over the Wi-Fi P2P connections. This is done with the help of the 
source node - a special device acting as a gateway between the CBTC and Wi-Fi P2P connections and running a client of the Peer-to-Peer (P2P) multimedia streaming software. Clients use the multimedia streaming software to request data from other clients and coordinate data deliver. This paper proposes to use the Peer-to-Peer Streaming Peer Protocol (PPSPP) for this purpose.

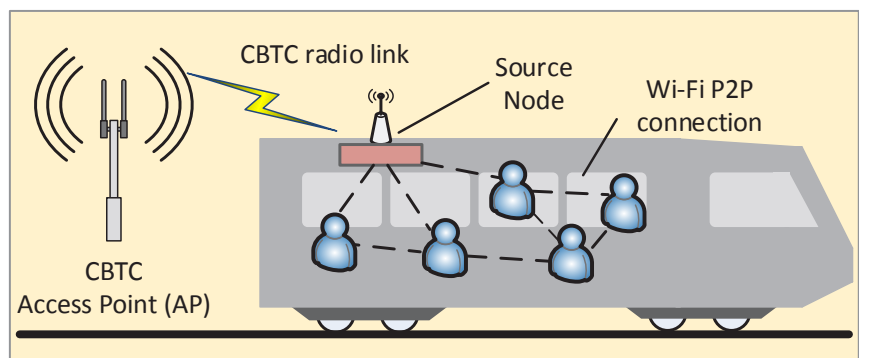

Fig. 2. An overview of the proposed multimedia streaming system.

The proposed multimedia streaming system addresses all the above listed technical challenges. As a CBTC radio communication network is designed to provide uninterrupted connectivity to the train, there are no areas without radio coverage. Furthermore, there are no data transmission interruptions when using the CBTC system compared to cellular connectivity as described previously.

The security of data communication withing the train car is ensured by employing encryption methods defined in the Wi-Fi P2P standard [4]. Furthermore, the Wi-Fi P2P programming interface supports programmatic setup of encrypted communication without user interaction [16]. Finally, using $\mathrm{P} 2 \mathrm{P}$ communication model makes each user acts as a proxy server. Once multimedia is delivered to the active user's device, other users can download it from there, instead of consuming bandwidth of the train's CBTC connection. It is important to note that each user is acting as a proxy only while it is streaming multimedia. As soon as user stops streaming multimedia, he leaves the P2P network to conserve the battery life of the device.

The work presented here is based on an early-stage system prototype described in [17], and includes the following updates. The system now uses updated data requesting and connections establishments algorithms ${ }^{1}$. These changes allow to establish connections between the user devices faster and to improve the video playback continuity. While the earlier work used network emulation alone to model the system, this work used real devices to verify that the system worked as intended.

Limited scale verification was performed using three "Raspberry Pi 3 Model B" devices that supported Wi-Fi P2P communication. Tests using "Raspberry Pi" devices were used to verify that a single Wi-Fi P2P device can maintain connections to multiple other devices and to evaluate the time required to setup a connection between two devices. Real devices were also used to measure connection bandwidth in multiple

\footnotetext{
${ }^{1}$ Annotated source-code available at github.com/justas-/PyPPSPP
}

operation modes. The results of these test were later used to calibrate the tests performed using a network emulator (see section IV for details).

Larger scale verification was done using a networks emulation software as described in section IV. Finally, the earlier work did not consider user dynamics. Test setup in the earlier work assumed that all users start multimedia streaming at the same time. This work considers dynamic user arrival and departure patterns, as seen in urban-transport systems. The remainder of the section provides a detailed description of above-listed technologies used to implement the multimedia streaming system.

\section{A. Communications-Based Train Control systems}

Conventional railway signaling systems, based on color light signals and trackside equipment such as track circuits, are rapidly being replaced by modern systems, widely known as communication-based signaling systems [18]. One of the most well-known of such systems is Communications-Based Train Control (CBTC) [19]. In communication-based signaling, a radio communication technology is used to transfer train control information between the train and the traffic control center. Currently there exist over 150 CBTC systems worldwide, including both operational and systems under-development [3]. Nearly all of these systems use IEEE 802.11 Wi-Fi [15] as the radio technology, mainly owing to its cost-effectiveness [3].

IEEE $802.11 \mathrm{a}$ or $802.11 \mathrm{~g}$ are the most widely used Wi-Fi technologies, supporting data rates of up to $54 \mathrm{Mbps}$. More advanced technologies such as 802.11n and 802.11ac support even higher rates, up to $1 \mathrm{Gbps}$. Using radio communication, CBTC systems enable transfer of real-time and high-resolution train control information between the train and the wayside. CBTC data does not impose high demands on the bandwidth. It consists of train control messages typically sent at intervals of about 500 milliseconds. Typical data rate ranges up to maximum $100 \mathrm{kbps}$ [3]. This leaves sufficient bandwidth for other purposes. However, in order to ensure safe operation of the CBTC system, the train-control data traffic should be strictly prioritized over other traffic types.

In a CBTC radio communication network, several Wi-Fi APs are deployed at the trackside and a train has to switch from one AP to another as it moves past them (i.e. perform a handover). To guarantee a continuous connectivity to the train, these APs are deployed in a way that their coverage areas overlap.

\section{B. Wi-Fi Peer-to-Peer}

Today, users like to interact with each other on their devices on a frequent basis, without the regard for the underlying communication technology. Thus, a sharp rise in the popularity of device-to-device (D2D) or Peer-to-Peer communication technologies is currently being witnessed. Although $802.11 \mathrm{Wi}-\mathrm{Fi}$ does support D2D communication with its infrastructure-less mode, also known as "ad-hoc mode", the feature has witnessed limited popularity owing to its lack of user-friendliness [10]. To enable more efficient means of D2D communication for 
Wi-Fi users, the Wi-Fi Forum adopted an extension to the IEEE 802.11 standard called Wi-Fi Peer-to-Peer (Wi-Fi P2P, also known as Wi-Fi Direct) [4]. It is worth noting that Wi-Fi $\mathrm{P} 2 \mathrm{P}$ is not an IEEE standard itself. Rather, it is a technical specification that defines the architecture and protocols to implement Wi-Fi based D2D communication. In Wi-Fi P2P, devices communicate in groups. In a group, a Wi-Fi P2P device may have the role of a Group Owner (GO) or of a client. The GO role provides group clients with AP-like functionalities and the GO is also occasionally referred to as a "Soft AP". The GO role is negotiated at the time of the group formation with the help of an election process. In a Wi-Fi group, the client devices may include both legacy (i.e. conventional 802.11 devices) and P2P clients [10]. It is also important to note, that all devices fully implementing the WiFi P2P standard can act as "Soft-AP".

A P2P device can be a part of a P2P group and maintain a conventional Wi-Fi connection simultaneously. Such a device is referred to as a P2P Concurrent Device [4], [9], [10]. This implies that for example in a $\mathrm{P} 2 \mathrm{P}$ group, a device can provide the group clients with a connection to the outer world by simultaneously connecting to a conventional Wi-Fi AP or a 3G/4G network. This, again, is a major advantage over a conventional ad-hoc network in which devices cannot simultaneously connect to existing Wi-Fi networks [4]. Thus, in contrast to a conventional Wi-Fi network in which a device can either operate as an AP or a client, in Wi-Fi P2P, these roles are arbitrary - any device can function as an AP. Furthermore, given that this new capability can be implemented entirely in software, without a need of making any changes to the hardware, bears a high significance [9].

\section{Peer-to-Peer Streaming Peer Protocol (PPSPP)}

The Wi-Fi P2P technology described earlier is used as a data carrier in the proposed multimedia distribution system. However, an additional protocol is needed to coordinate the multimedia data exchange between the users of the system. There is already a large number of such protocols proposed [20] in the field of P2P multimedia streaming. The work presented here uses the Peer-to-Peer Streaming Peer Protocol (PPSPP) [5] as it is the only P2P communication protocol designed for multimedia streaming and standardized by the Internet Engineering Task Force.

PPSPP works by dividing multimedia data into a number of fixed-size pieces called chunks. Each chunk is uniquely identified by a chunk number. Each user participating in multimedia data exchange keeps track of the chunks it has already downloaded. Periodically, each user informs all other connected users about the chunks it has by sending a HAVE message containing a list of chunks. After receiving a HAVE message from a user, the recipient compares a list of chunks available locally to that in the HAVE message. If the remote user advertises chunks that are not available locally, the recipient of the HAVE message requests the missing chunks by sending a REQUEST message to the advertising user. After receiving a request for data, an advertising user sends the requested chunks using DATA messages.

As the user is streaming multimedia, it keeps all downloaded chunks on own device and makes them available to other users. This method of keeping a copy of data and sharing it with other users allows to significantly reduce the load on the upstream Internet connection because new users requesting multimedia data will first download them from other users' devices.

\section{EXPERIMENTAL EVALUATION}

\section{A. Experimental Setup}

The performance evaluation of the multimedia streaming systems was performed in a virtual environment using the CORE network emulator [21]. CORE divides the workstation running experiments into a number of virtual instances. Each instance represents one user performing multimedia streaming over Wi-Fi P2P using a PPSPP software client. The physical and media access layers of Wi-Fi connections were emulated using EMANE emulator, a part of the CORE software. The Wi-Fi MAC layer used the IEEE 802.11g standard with the maximum data rate set to 54 Mbps. During the experiments, clients were placed at uniformly random coordinates within an area of $3.6 \times 10.5$ meters, following the dimensions of a train car of the Copenhagen S-train network. The experimental setup considers a single train car in order to reduce the experiment run-time. Systems deployed in actual trains would use one source-node per train car to increase the system capacity. In all scenarios, the multimedia record is a 1280x720 (HD 720p) video clip encoded with a VP8 codec having a bitrate of $2 \mathrm{Mbps}$. The length of the record was 5 minutes.

In order to evaluate the quality characteristics of streaming multimedia during users turnover, experiments were designed as follows. At the start of each experiment, a number of users started multimedia streaming using the proposed system. The experiments were repeated with 15, 20 and 25 starting users. Then after a period of time (3, 5 or 7 minutes), either extra users were added (Add scenarios) or a part of users was replaced with new users (Replace scenarios). The number of users added or replaced varied between zero and $80 \%$ of the starting population. After adding or replacing users, the experiment continued for the same period of time. Such experiment setup allowed to measure what impact the adding or replacing of users, at regular time intervals, has on the streaming multimedia's quality. The scenarios considering removal of users are not included in this work, as results described next indicate that removing users does not affect the streaming multimedia's quality negatively.

Overall, this work considers 90 different combinations of parameters. Simulations using each unique combination (scenario) of parameters were repeated 5 times and results averaged.

The previous work done in the field of streaming multimedia to public transport [6]-[8] mainly considers the parameters of network or transport layer, such as average throughput or packet loss. This work, in contrast, focuses specifically on the QoS parameters of the multimedia streams received by the 


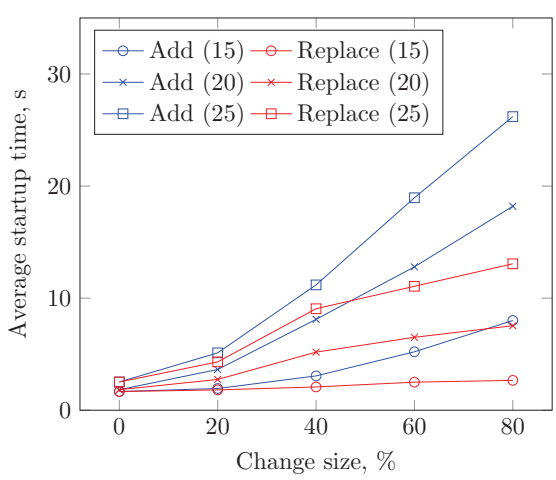

(a) 3-minute interval

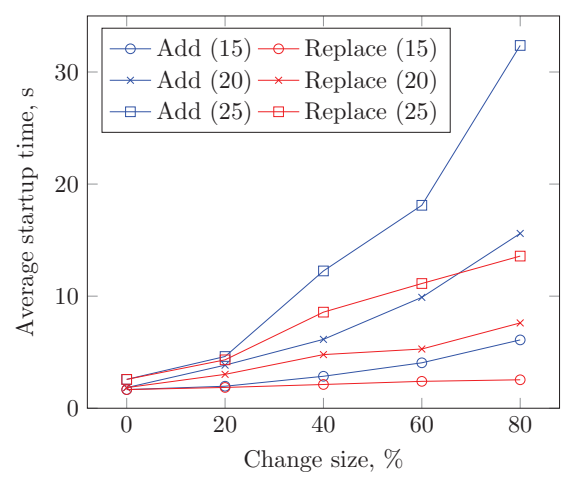

(b) 5-minute interval

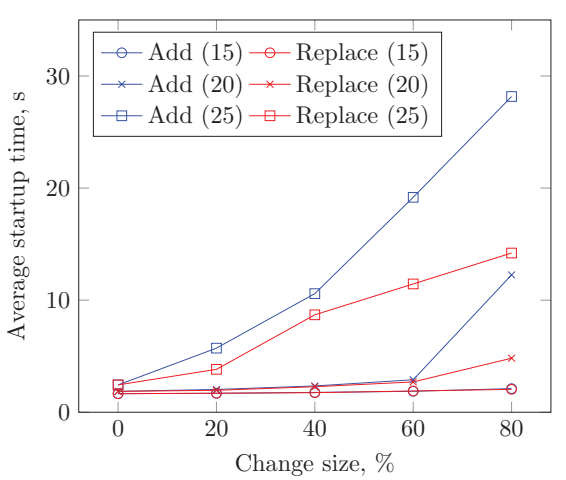

(c) 7-minute interval

Fig. 3. Average observed user start-up times. Numbers in the parentheses in the legends indicate the starting users population.

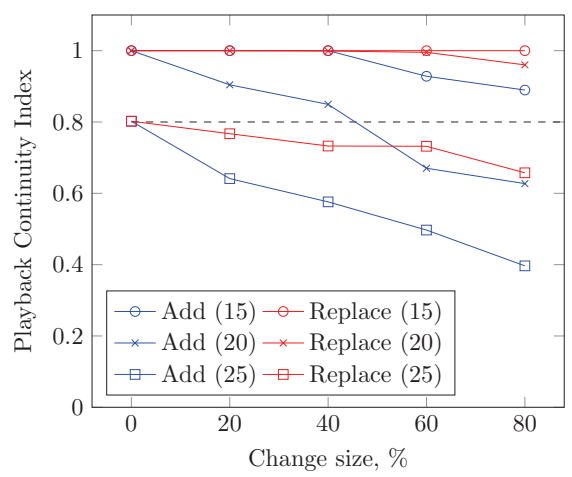

(a) 3-minute interval

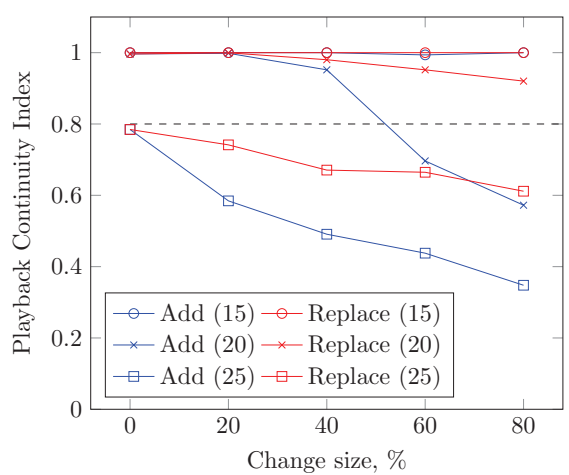

(b) 5-minute interval

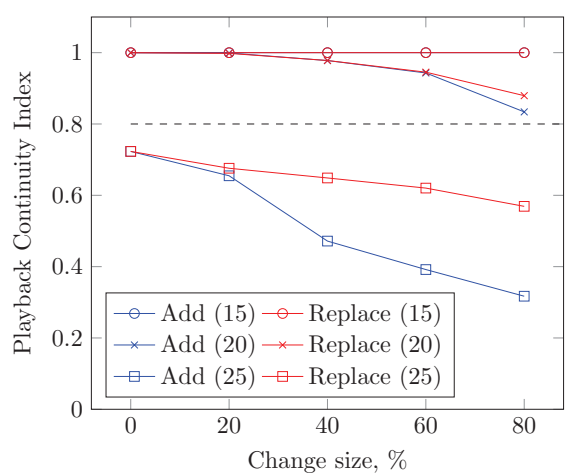

(c) 7-minute interval

Fig. 4. Average observed Playback Continuity Index (PCI). Numbers in the parentheses in the legends indicate the starting user population. Dashed lines indicate the chosen performance threshold.

system users. Specifically, experiments in this paper consider two QoS parameters - start-up time of multimedia stream and the Playback Continuity Index (PCI). The start-up time, refers to the time period from starting the streaming application to the time the rendering of multimedia stream starts. The playback continuity index is the ratio of the number of rendered video frames in the user's device to the number of attempts to render the next frame. The PCI value can range from 0 (no video frames were rendered in time) to 1 (all frames were rendered in time). From the point of view of user's experience, PCI quantifies how "choppy" (stopping to wait for data and then resuming) the rendering process is. The PCI value of 0.8 was chosen as the lowest acceptable quality level.

\section{B. Experimental results - Start-up time}

Results for the average client start-up times are shown in Fig. 3. Here, each sub-figure shows the average start-up times for each of the 3,5, and 7 minute time intervals before and after user population changes. Comparing the results between the "add" and "replace" scenarios, it can be seen that clients in the "add" scenarios experienced longer start-up times than clients in the "replace" scenarios. This is especially visible in the 3 minutes case (Fig. 3a). In this example, when the number of users increases from 20 to 32 (change size of $60 \%$ ), the multimedia playback started after 6.5 seconds in the "replace" scenario, and 12.8 seconds in the "add" scenario.

The longer start-up times in the "add" scenarios are caused by the increase in the number of active users. In the "replace" scenarios, the total number of active users stays unchanged $(15,20$ or 25 users), while in the "add" scenarios, the total number of users increases with the change size. As all users communicate using the same Wi-Fi radio channel, a higher number of users introduce more interference, thus reducing the effective transmission speed and increasing the time required to fill the playback buffer.

By comparing the results for the different time intervals, it can be seen that the average start-up time is similar when the interval is 3 and 5 minutes (Fig. 3a and 3b), but decreases significantly when the time interval is 7 minutes (Fig. 3c). The reduction of the average start-up time here is the result of the chosen video file length of 5 minutes. In a test scenario with the 7 minutes time interval, all users in the system are able to download the file fully before new users arrive. When the new users arrive, they are able to request data from multiple users concurrently, thus reducing the time required to fill the initial playback buffer. For comparison, users in 3 and 5 minutes 
scenarios are still downloading the multimedia file when the new users arrive.

\section{Experimental results - Playback Continuity Index (PCI)}

The average observed PCI values for different time periods between the stops are shown in Fig. 4. For all time periods, the results indicate that the system performs within the chosen quality threshold (PCI $>0.8$ ) when the starting user population is 25 users. This observation is valid for both "add" and "replace" scenarios. Furthermore, in all tests using different time intervals, the "add" scenarios resulted in worse average PCI values than the "replace" scenarios. As with the start-up time results discussed previously, this is caused by the radio interference created by the users operating on the same Wi-Fi channel, thus reducing the effective data transfer speeds.

Comparing the results for the different time intervals, it can be seen that the average PCI values for the 7 minutes scenario (Fig. 4c) are higher than those of the 3 minutes scenario (Fig. 4a). This is especially visible in the "add" scenarios. For example, when the number of users changes from 20 to 36 ( $80 \%$ increase), the PCI value is 0.62 in 3 minutes scenario, and 0.83 in 7 minutes scenario. This indicates that the quality of multimedia is negatively impacted by the frequent change in users population.

Overall, the obtained results indicate two main outcomes. First, the proposed multimedia streaming system delivers multimedia within the chosen QoS threshold when the number of concurrent users is less than 25. For the urban-trains use-case, this means that each train car should have at least one source node to serve the passengers. Second, the system delivers higher quality multimedia when the time intervals between the stops are longer. This can limit the deployment of the system in railway lines with frequent stops.

\section{CONCLUSIONS}

This work presents a multimedia distribution system that can be used to distribute streaming data to urban train passengers. The data is delivered to the train using the available capacity of a CBTC radio communication link and subsequently distributed to the passengers using Peer-to-Peer (P2P) streaming over Wi-Fi P2P (Wi-Fi Direct) connections. Using Wi-Fi P2P as a data carrier allows for easier deployment in transport infrastructure as users can maintain concurrent connections to other Wi-Fi P2P devices and the conventional AP-based Wi-Fi networks.

The experimental evaluation of the proposed system indicates that the system can support up to 25 concurrent users while delivering high-quality multimedia. The effective number of users can be increased by employing one such system per train car. However, the performance of multimedia distribution is affected by user turnover. As users turnover is unavoidable in trains, such system would be more suitable for train lines with fewer stops and with longer time intervals between the stops.

\section{REFERENCES}

[1] "Ericsson Mobility Report, June 2017," Tech. Rep., 2017. [Online]. Available: ericsson.com/mobility-report

[2] D. T. Fokum and V. S. Frost, "A survey on methods for broadband internet access on trains," IEEE communications surveys \& tutorials, vol. 12, no. 2, pp. 171-185, 2010.

[3] J. Farooq and J. Soler, "Radio communication for communications-based train control (CBTC): a tutorial and survey," IEEE Communications Surveys \& Tutorials, vol. 19, no. 3, pp. 1377-1402, 2017.

[4] "Wi-Fi Peer-to-Peer (P2P) Technical Specification Version 1.7," Tech. Rep., 2016. [Online]. Available: https://www.wi-fi.org/discover-wifi/specifications

[5] A. Bakker, R. Petrocco, and V. Grishchenko, "Peer-to-Peer Streaming Peer Protocol (PPSPP)," RFC 7574, Jul. 2015.

[6] H.-T. Chiao, S.-Y. Chang, K.-M. Li, Y.-T. Kuo, and M.-C. Tseng, "Wi$\mathrm{Fi}$ multicast streaming using AL-FEC inside the trains of high-speed rails," in Broadband Multimedia Systems and Broadcasting (BMSB), 2012 IEEE International Symposium on. IEEE, 2012, pp. 1-6.

[7] E. Hernandez and A. Helal, "Examining mobile-ip performance in rapidly mobile environments: the case of a commuter train," in Local Computer Networks, 2001. Proceedings. LCN 2001. 26th Annual IEEE Conference on. IEEE, 2001, pp. 365-372.

[8] L. Zhu, F. R. Yu, B. Ning, and T. Tang, "Cross-layer design for video transmissions in metro passenger information systems," IEEE Transactions on Vehicular Technology, vol. 60, no. 3, pp. 1171-1181, 2011.

[9] D. Camps-Mur, A. Garcia-Saavedra, and P. Serrano, "Device-to-device communications with Wi-Fi Direct: overview and experimentation," IEEE wireless communications, vol. 20, no. 3, pp. 96-104, 2013.

[10] M. Conti, F. Delmastro, G. Minutiello, and R. Paris, "Experimenting opportunistic networks with Wi-Fi Direct," in 2013 IFIP Wireless Days (WD). IEEE, nov 2013, pp. 1-6. [Online]. Available: http://ieeexplore.ieee.org/document/6686501/

[11] A. Detti, B. Ricci, and N. Blefari-Melazzi, "Mobile peer-to-peer video streaming over information-centric networks," Computer Networks, vol. 81 , pp. 272-288, 2015 .

[12] A. Pyattaev, O. Galinina, K. Johnsson, A. Surak, R. Florea, S. Andreev, and Y. Koucheryavy, "Network-assisted D2D over Wi-Fi Direct," in Smart Device to Smart Device Communication. Springer, 2014, pp. $165-218$.

[13] O. B. Karimi, J. Liu, and C. Wang, "Seamless wireless connectivity for multimedia services in high speed trains," IEEE Journal on selected areas in communications, vol. 30, no. 4, pp. 729-739, 2012.

[14] S.-Y. Chang, H.-T. Chiao, X.-Y. Yeh, and M.-C. Tseng, "UDP-based file delivery mechanism for video streaming to high-speed trains," in Personal Indoor and Mobile Radio Communications (PIMRC), 2013 IEEE 24th International Symposium on. IEEE, 2013, pp. 3568-3572.

[15] Wireless LAN Medium Access Control (MAC) and Physical Layer (PHY) Specifications, IEEE Std. 802.11, 2012.

[16] WifiP2pConfig — Android Developers. Accessed: 2017-10-01. [Online]. Available: https://developer.android.com/reference/android/net/wifi/p2p/ WifiP2pConfig.html

[17] J. Poderys, J. Farooq, and J. Soler, "A multimedia streaming system for urban rail environments," in International Workshop on Communication Technologies for Vehicles. Springer, 2017, pp. 41-53.

[18] Frost \& Sullivan. (2013, Jul.) Strategic analysis of communication based train control systems in the western European urban rail market. [Online]. Available: http://www.frost.com/sublib/display-report.do?id= M92D-01-00-00-00

[19] Communications-Based Train Control (CBTC) Performance and Functional Requirements, IEEE Std. 1474.1, 2004.

[20] M. Hosseini, D. T. Ahmed, S. Shirmohammadi, and N. D. Georganas, "A survey of application-layer multicast protocols," IEEE Communications Surveys \& Tutorials, vol. 9, no. 3, pp. 58-74, 2007.

[21] J. Ahrenholz, "Comparison of CORE network emulation platforms," Proceedings - IEEE Military Communications Conference MILCOM, pp. 166-171, 2010. 